\title{
Theoretical Investigation on the Performance of Multicomponent Distillation Column for the Separation of Hydrocarbon Mixture Using Inside out Approach
}

\author{
Shashwat Bansal and Sampatrao D. Manjare
}

\begin{abstract}
In this paper, the performance of a distillation column with a multicomponent hydrocarbon mixture as a feed is theoretically studied. The rigorous solution technique namely the inside out method has been used to predict the performance of the system under study. The mathematical model of the inside out method has been developed and detailed simulation studies have been carried out to predict the performance of the separation of multicomponent hydrocarbon mixture. In simulation studies, temperature profile, composition profile and flow rates of both liquid and vapor phases has been computed. The column profiles computed using inside out method have been compared with the profiles obtained using bubble point method, LewisMatheson method and a SCDS column in CHEMCAD process simulator. The effect of feed location on the column performance has been studied and an optimum feed location has been determined. The condenser and reboiler duties for the column have also been calculated. The convergence behavior of the inside out method has also been studied by plotting a profile of its convergence parameters.
\end{abstract}

Index Terms-Equilibrium stage distillation, hydrocarbon separation, inside out method, multicomponent mixture, twotier algorithm.

\section{INTRODUCTION}

Distillation is one of the popular separation technologies and its application extends to a wide variety of process industries. Theoretical investigation on the performance of multicomponent distillation column is the basis for its design and development. The rigorous methods such as the Lewis-Matheson (LM) method [1], the Thiele- Geddes (TG) method [2] are used for rigorous calculation of multicomponent separation using multistage distillation column. These methods are based on equation tearing procedures. Friday and Smith [3] studied various equation tearing procedures and revealed that there is no single equation tearing procedure which could solve different kinds of problems. Friday and Smith [3] further suggested that bubble point method and sum rates methods be used for narrow boiling and wide boiling feeds respectively. Boston \& Sullivan [4] and Naphtali \& Sandholm [5] have proposed more robust methods viz inside out method and Newton Raphson method respectively and are useful to solve complicated models.

Manuscript received June 27, 2015; revised October 29, 2015.

The authors are with the Department of Chemical Engineering, BITSPilani, K.K. Birla, Goa Campus, Goa 403726, India (corresponding author: Sampatrao D. Manjare; tel.: +91-8322580112; fax: +91-8322557030; email:manjare@goa.bits-pilani.ac.in,shashwat56@gmail.com)
Suitable choice of iteration variable in the inside out method results in relatively small number of model equations and hence require less computer memory and computation time as compared to Newton Raphson method. Simandl and Svrcek [6] present a comparison of convergence time required for inside out method and Newton Raphson method. The analysis reveals that inside out method has a faster convergence time.

Therefore from above discussion, it is clear that inside out approach is the preferred choice for simulating multistage liquid vapor separators and is considered for study in this paper.

To understand the detailed state of the art of the inside out method, literature survey has been carried out and presented below.

Boston and Sullivan [4] proposed a new algorithm for steady state solution of the equations that describe multicomponent, multistage separation processes. The algorithm has been found to be exceptionally stable and efficient. Boston [7] discussed the challenges faced in solving models using class I methods namely bubble point and sum rates method and class II methods namely Newton Raphson method. Subsequently a detailed simulation approach of inside out method for multicomponent multistage liquid vapor separator has been presented. Russell [8] suggested changes to the inside out method by using a quasi-Newton approach to achieve all enthalpy balance and performance specifications directly. Saeger and Bishnoi [9] suggested a modified inside out algorithm for highly non ideal liquid solutions using a two parameter activity coefficient model. Jelinek [10] extends the methods suggest by Russell [8] by providing a detailed methodology and discusses certain advantages and potential shortcomings. Shan et al. [11] suggests a method to efficiently solve tridiagonal matrices containing off band elements. These suggestions make solving columns with side strippers and pump around much easier.

From above mentioned literature survey it is clear that researchers across the world are striving to simplify the inside out algorithm to be used for the simulation of multicomponent distillation column.

\section{INSIDE OUT METHOD}

The inside out method was first presented by Boston and Sullivan [4] as a new class of method for solving multistage multicomponent distillation. It was initially applicable only for the separation of an ideal hydrocarbon feed but its capabilities were greatly extended to various complicated 
systems as studied by Russell [8], Jelinek [10], Simandl \& Svrcek [6], Trevino \& Kisala [12], Saeger and Bishnoi [9] and Shan et al. [11]. The inside out method can currently solve for any multicomponent multistage liquid vapor separation like distillation, absorption, stripping and reboiled absorption. The method is very robust and permits a range of allowable specifications. Also the method has been found to be insensitive of initial estimates and exceptionally stable. The method is known to converge for difficult separation problems where traditional methods like Thiele-Geddes [2] and Lewis-Matheson [1] fail and is known to be just as efficient as any other technique while solving simple columns.

To further understand the methodology of inside out method, a mathematical model of a single stage liquid vapor separator at steady state has been presented below.

\section{A. The Mathematical Model}

The model presents a cascade of $N$ counter current stages with the following assumptions

1) All stages are equilibrium stages.

2) Kinetic and potential energy changes in the system are ignored.

3) There is no entrainment of liquid or trapping of bubble vapor in liquid.

Diagram of a single stage out of $N$ stages in the cascade is given in Fig. 1 below.
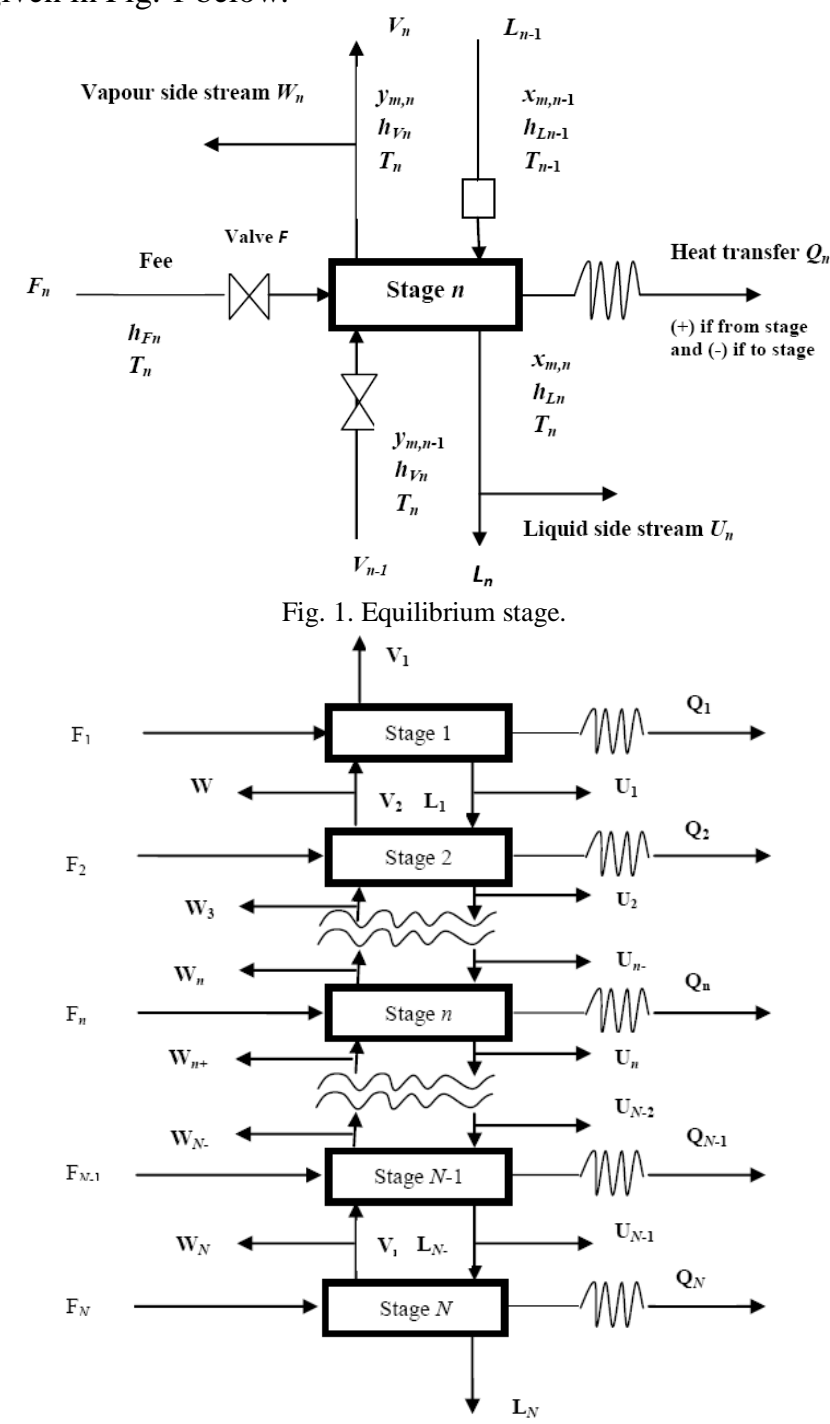

Fig. 2. Cascade of $\mathrm{N}$ equilibrium stages.

\section{MESH Equations:}

The model has been developed by writing materialbalance, energy-balance, summation relations and equilibrium relations for each stage. These equations are collectively known as the MESH equations. The MESH equation for inside out method, presented here uses volatility and stripping factors along with enthalpy to model a steady state continuous liquid vapor separator. The diagram of a cascade of $\mathrm{N}$ stages has been given in Fig. 2 below for a continuous steady state separator.

The following variables are defined to be used in the MESH equations

Relative volatility: $\quad \alpha_{m, n}=\frac{K_{m, n}}{K_{b, n}}$

Base component stripping factor: $\quad S_{b, n}=\frac{K_{b, n} * V_{n}}{L_{n}}$

Withdrawal factor for liquid phase: $R_{L_{n}}=1+\frac{U_{n}}{L_{n}}$

Withdrawal factor for vapor phase: $R_{v_{n}}=1+\frac{W_{n}}{V_{n}}$

The MESH equations to be used for the inside out method are as follows:

Material balance for each component at each stage $(M-$ equations)

$$
\begin{aligned}
& -f_{m, n}= \\
& l_{m, n-1}-\left(R_{L_{n}}+\alpha_{m, n} S_{b, n} R_{v_{n}}\right) l_{m, n}+\left(\alpha_{m, n+1} S_{b, n+1}\right) l_{m, n+1}
\end{aligned}
$$

Phase equilibrium relations at each stage (E-equations)

$$
v_{m, n}=\alpha_{m, n} S_{b, n} l_{m, n}
$$

Summations of component liquid and vapor flow (Sequations)

$$
\begin{aligned}
& V_{n}=\sum_{m=1}^{c} v_{m, n} \\
& L_{n}=\sum_{m=1}^{c} l_{m, n}
\end{aligned}
$$

Energy/Enthalpy balance at each stage(H-equations)

$$
\begin{gathered}
H_{n}=h_{L_{n}} R_{L_{n}} L_{n}+h_{V_{n}} R_{v_{n}} V_{n}-h_{L_{n-1}} L_{n-1}-h_{V_{n+1}} V_{n+1}- \\
h_{F_{n}} F_{n}-Q_{n}=0
\end{gathered}
$$

\section{B. Simulation Studies Using Inside out Method}

Fig. 3 presents the proposed solution methodology for inside out method. The initialization procedure to obtain estimates is similar to bubble point [13] method and sum rates [14] methods.

\section{Operating Mode}

In order to test the model presented, a simulation has been run on the problem statement given below by writing a $\mathrm{C}$ code. A damping factor of 1.2 has been used to accelerate convergence. The results obtained are compared with Lewis-Matheson method [1] and bubble point method [13] and process simulator CHEMCAD to validate the model. The problem statement is taken from Smith [15].

The study has been carried out with 11 bubble cap tray column and a partial reboiler. Consequently, the number of 
corresponding theoretical trays is 10 with a feed at middle tray. The column has the maximum top vapor capacity of 1.75 times the volume of feed to be handled. The column operates at the pressure of 120 psia. The feed is at its bubble point and has the average composition provided in Table I. The $\mathrm{D} / \mathrm{F}$ ratio is given as 0.489 .

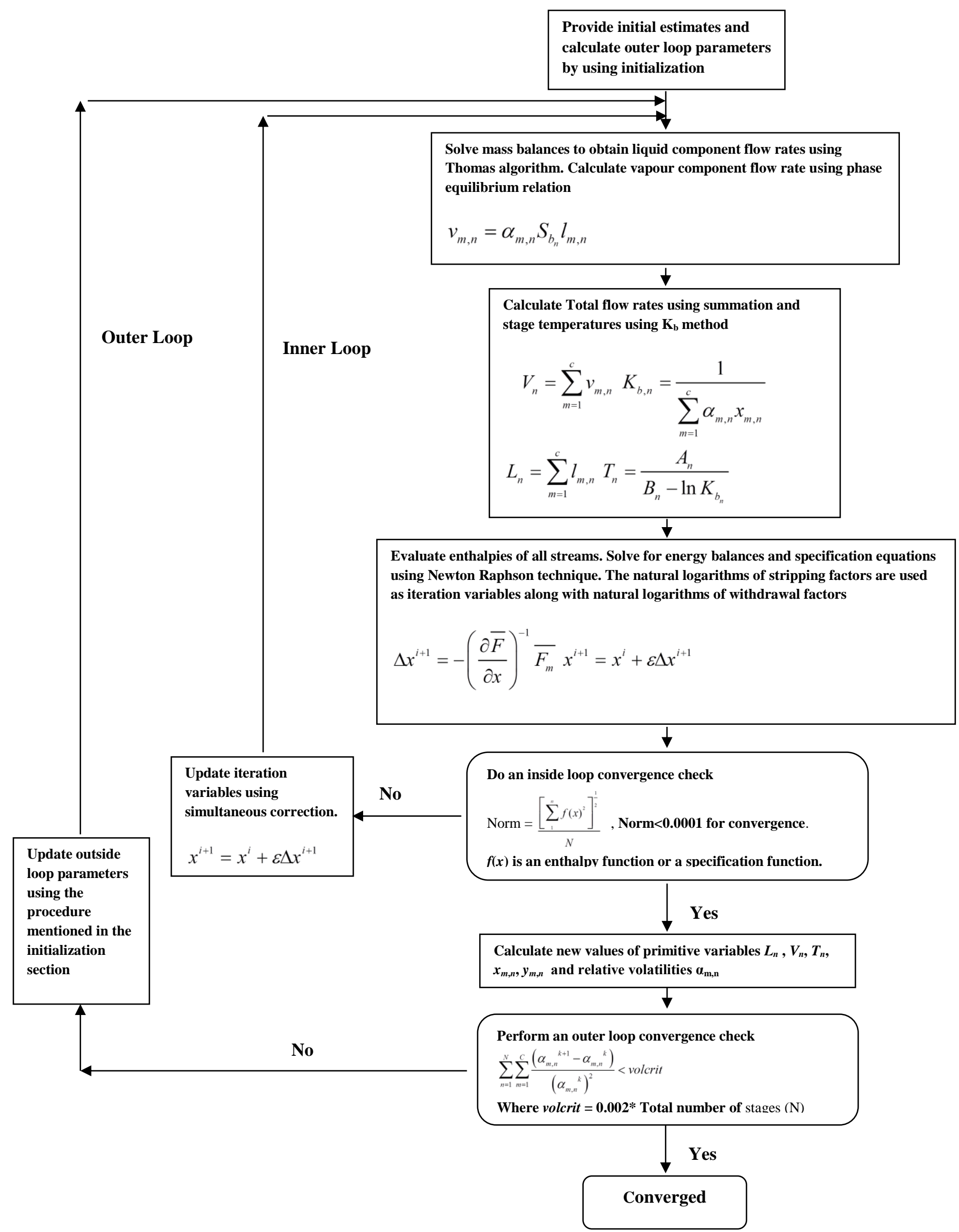

Fig. 3. Proposed flowchart for inside out method. 
TABLE I: AVERAGE COMPOSITION OF THE FEED

\begin{tabular}{|c|c|c|c|c|c|c|}
\hline Component & $\mathrm{C}_{3}$ & $i \mathrm{C}_{4}$ & $n \mathrm{C}_{4}$ & $i \mathrm{C}_{5}$ & $n \mathrm{C}_{5}$ & Total \\
\hline $\mathrm{Fx}_{\mathrm{F}}$ & 5 & 15 & 25 & 20 & 35 & 100 \\
\hline
\end{tabular}

Further it is mentioned that column will only be operated ifless than $7 \mathrm{~mol} \% \quad i-\mathrm{C}_{5}$ is present in the overhead and less that $3 \mathrm{~mol} \% n-\mathrm{C}_{4}$ is present in the bottoms.

\section{1) Expressions for K-values and enthalpy}

The expressions used for K-values and enthalpy have been obtained by fitting values from data tables over a range of temperatures and at a given pressure

2) Expression for $K$ values

$$
\begin{gathered}
K(\text { propane })=(0.00007 * \mathrm{~T} * \mathrm{~T})-(0.003 * \mathrm{~T})+1.441 \\
K(i \text {-butane })=(0.00005 * \mathrm{~T} * \mathrm{~T})-(0.002 * \mathrm{~T})+0.531 \\
K(n \text {-butane })=(0.00005 * \mathrm{~T} * \mathrm{~T})-(0.006 * \mathrm{~T})+0.745 \\
K(i \text {-pentane })=(0.00003 * \mathrm{~T} * \mathrm{~T})-(0.004 * \mathrm{~T})+0.438 \\
K(n \text {-pentane })=(0.00003 * \mathrm{~T} * \mathrm{~T})-(0.004 * \mathrm{~T})+0.327
\end{gathered}
$$

3) Expression for liquid enthalpy

$$
\mathrm{H}_{\mathrm{l}}(\text { propane })=((0.061 * \mathrm{~T} * \mathrm{~T})+(18.43 * \mathrm{~T})+5056) * 2.2 * 1.055
$$$$
\mathrm{H}_{\mathrm{l}}(i \text {-butane })=((0.045 * \mathrm{~T} * \mathrm{~T})+(24.31 * \mathrm{~T})+5938) * 2.2 * 1.055
$$$$
\mathrm{H}_{1}(n \text {-butane })=((0.027 * \mathrm{~T} * \mathrm{~T})+(30.12 * \mathrm{~T})+5963)
$$$$
* 2.2 * 1.055
$$$$
\mathrm{H}_{\mathrm{l}}(i \text {-pentane })=((0.047 * \mathrm{~T} * \mathrm{~T})+(28.61 * \mathrm{~T})+7237)
$$$$
* 2.2 * 1.055
$$

$$
\mathrm{H}_{\mathrm{l}}(n \text {-pentane })=((0.049 * \mathrm{~T} * \mathrm{~T})+(29.17 * \mathrm{~T})+7438)
$$

$$
* 2.2 * 1.055
$$

\section{4) Expression for vapor enthalpy}

$$
\mathrm{H}_{v}(\text { propane })=((-0.009 * \mathrm{~T} * \mathrm{~T})+(27.74 * \mathrm{~T})+11478)
$$

$* 2.2 * 1.055$

$\mathrm{H}_{v}(i$-butane $)=((0.039 * \mathrm{~T} * \mathrm{~T})+(11.98 * \mathrm{~T})+14902) * 2.2 * 1.055$

$\mathrm{H}_{v}(n$-butane $)=((0.045 * \mathrm{~T} * \mathrm{~T})+(8.686 * \mathrm{~T})+16582) * 2.2 * 1.055$

$\mathrm{H}_{v}(i$-pentane $)=((0.063 * \mathrm{~T} * \mathrm{~T})+(4.127 * \mathrm{~T})+20458)$

$$
* 2.2 * 1.055
$$

$\mathrm{H}_{v}(n$-pentane $)=((0.00 * \mathrm{~T} * \mathrm{~T})+(28 * \mathrm{~T})+19300) * 2.2 * 1.055$

where temperature $\mathrm{T}$ is in ${ }^{\circ} \mathrm{F}$ and liquid and vapor enthalpy $\mathrm{H}_{1}$ and $\mathrm{H}_{v}$ are in $\mathrm{J} / \mathrm{mol}$.

\section{SOLUTION PROCEDURE}

The model equations were solved for by writing a $\mathrm{C}$ code. The material balances were solved using Thomas algorithm and the energy balances were solved using simultaneous correction. The results obtained are vapor flow rate, liquid flow rate, temperature and composition of both phases at each stage along with condenser and reboiler duties. The model required three outer loop iterations to converge.

\section{A. Convergence Criteria for Inner and Outer Loop}

Table II presents the convergence criteria for inner and outer loop. The inner loop convergence is based on how close to zero the values of enthalpy and discrepancy are at each stage while outer loop converges is based on closeness of values of relative volatilities between two successive iterations.

TABLE II: CONVERGENCE CRITERIA FOR INNER AND OUTER LOOP

TABLE II: CONVERGENCE CRITERIA FOR INNER AND OUTER LOOP
\begin{tabular}{|l|l|}
\hline Inner loop & \multicolumn{1}{c|}{ Outer loop } \\
\hline norm $=\frac{\left|\sum_{1}^{n} f(x)^{2}\right|^{1 / 2}}{N}{ }^{0.0001}[16]$ & $\begin{array}{l}\sum_{n=1}^{N} \sum_{m=1}^{c} \frac{\left(\alpha_{m, n}{ }^{k+1}-\alpha_{m, n}{ }^{k}\right)}{\left(\alpha_{m, n}{ }^{k}\right)^{2}}< \\
\text { volcrit }[6]\end{array}$ \\
$\begin{array}{l}\text { where } f(x) \text { is either the } \\
\text { enthalpy function } H_{n} \text { or the } \\
\text { discrepancy function } D_{1} \text { and } \\
D_{2} .\end{array}$ & $\begin{array}{l}\text { where volcrit }=0.002 * \text { Total number } \\
\text { of stages }(N)\end{array}$ \\
\hline
\end{tabular}

$\alpha_{m, n}$ is the relative volatility of components with respect to a base component.

\section{RESUlTS AND DISCUSSION}

This section provides the column profiles for temperature, vapor \& liquid composition and flow rates along with inner and outer loop convergence parameters. Further it also compares the result obtained from inside out approach with results obtained using bubble point method [13] and Lewis Matheson method [1]. Simulation results from a process simulator namely CHEMCAD has also been compared with inside out results. All the results are presented in the section below.

\section{A. Profile of Inner and Outer Loop Convergence Parameters}

Fig. 4 presents the profile for the inner loop convergence parameter (Norm). It is noted from Fig. 4, that after the first iteration, a steep drop is observed in the Norm value, followed by its gradual decrease. As mentioned by Simandl and Svrcek [6] the inner loop convergence exhibits creeping as it approaches the final solution.

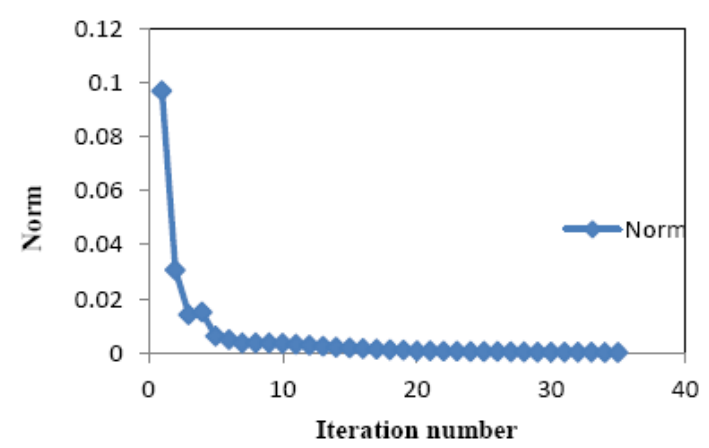

Fig. 4. Inner loop convergence profile.

Fig. 5 presents the profile for the outer loop convergence parameter (Volcrit). The outer loop are used to update parameters used by the inside loop for solving MESH equations. It can be observed that the outer loop is run infrequently as compared to the inner loop.

\section{B. Temperature Profile in the Column}

Fig. 6 shows the comparison between the simulated stage-wise temperatures and initially assumed temperatures. At the feed stage, a little change in the simulated profile is observed. This may be attributed to the fact that entering feed affects the temperature profile at feed stage. This is a 
commonly observed phenomenon. The profile exhibits temperature increase towards the bottom of the column. Fig. 7 presents the comparison of temperature profiles computed using various methods. From Fig. 7 it is noted that the inside out temperature profile is in accordance with the temperature profiles predicted by Lewis Matheson and CHEMCAD process simulator. Further it is also noted that the predictions by bubble point method are not in good agreement by rest of the three methods.

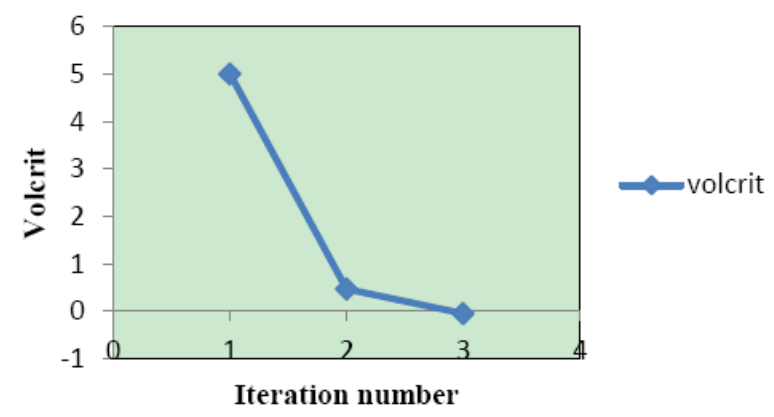

Fig. 5. Outer loop convergence profile.

This may be attributed to the fact that the mixture under study includes marginally wide range of boiling components and bubble point method is more suited to simulate systems containing narrow boiling range components.

\section{Liquid and Vapor Flow Rate Profile in the Column}

The liquid and vapor profile is presented in Fig. 8 and Fig. 9 respectively. From the Fig. 8 and Fig. 9 it is observed that both liquid and vapor flow rates decrease from the top stage towards the feed stage (from $1^{\text {st }}$ to $6^{\text {th }}$ stage). This implies that the assumption of constant inter-stage molar flow rates does not hold true in the rectifying section.

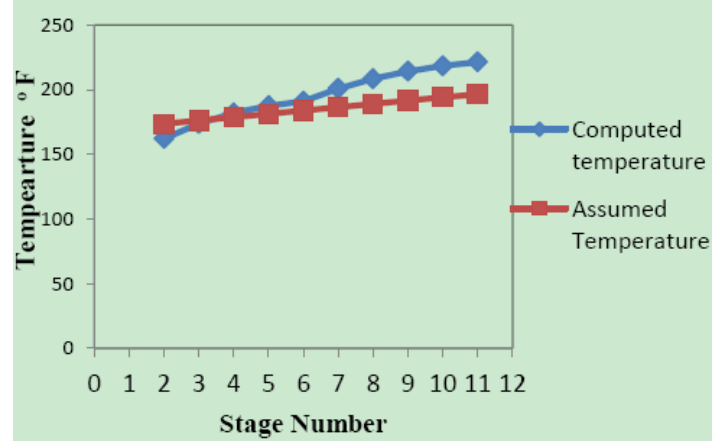

Fig. 6. Assumed and computed stage wise temperature profile.

Further it is observed that, sudden increase of the liquid flow rate occurs across the feed stage by an approximately equal amount to the feed rate because of the introduction of the feed as a saturated liquid. Since the feed is a saturated liquid the vapor rate changes slightly across the feed stage.

Liquid and vapor profile computed using inside out method has been compared with the profiles obtained using bubble point method and CHEMCAD process simulator and are presented in Fig. 10 and Fig. 11 respectively.

From the figures it is noted that the liquid and vapor profile obtained using inside out method is in good agreement with the profile obtained using CHEMCAD simulator but not with the profile obtained using bubble point method. This may be attributed to the reasons explained in the section above.

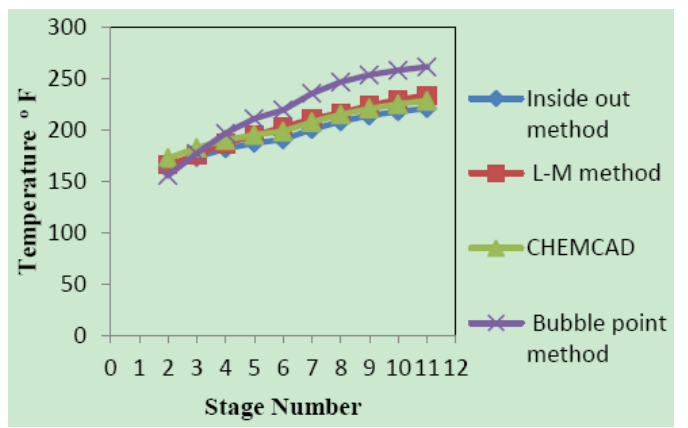

Fig. 7. Comparison of stage wise temperature profiles with other methods.

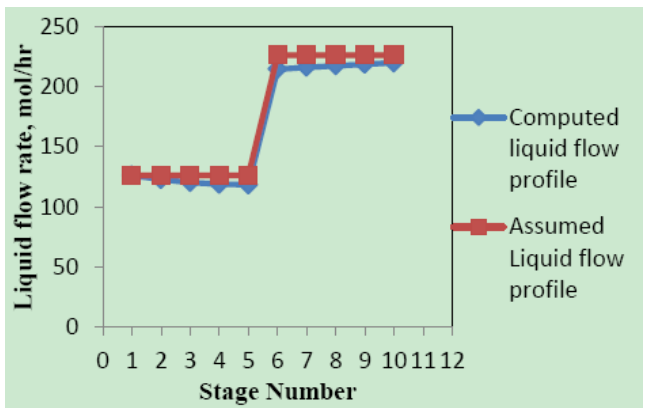

Fig. 8. Liquid flow rate profile in the column using inside out method.

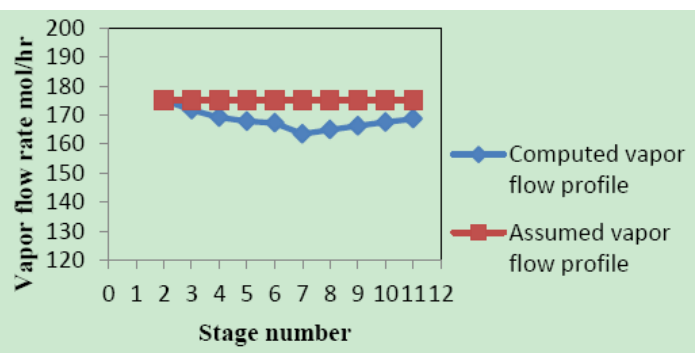

Fig. 9. Vapor flow rate profile in the column using inside out method.

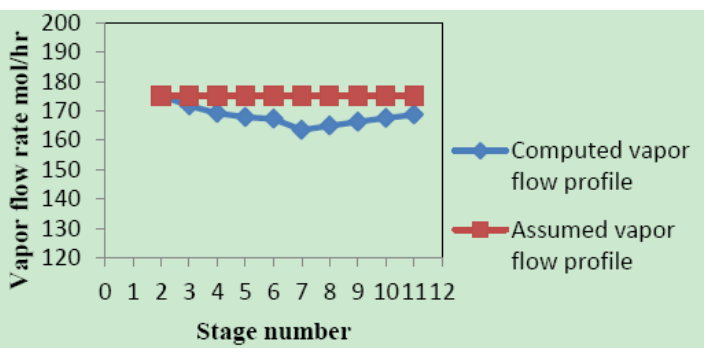

Fig. 10. Comparison of liquid flow rate profile in the column with other methods.

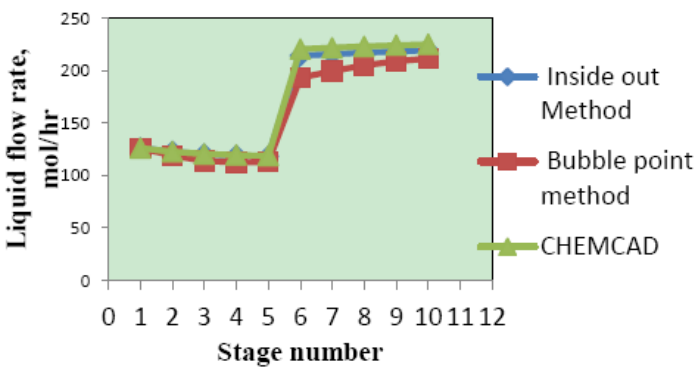

Fig. 11. Comparison of vapor flow rate profile computed using inside out with other methods.

\section{Liquid Composition Profile in the Column}

As mentioned in the problem statement, the column is a butane pentane splitter and thus separation is between two key components viz. $n-\mathrm{C}_{4}$ and $i-\mathrm{C}_{5}$. Thus, these two components can be designated as the light key (LK) and heavy key (HK), respectively. Thus $\mathrm{C}_{3}$ and $i-\mathrm{C}_{4}$ are lighter- 
than-light key (LLK), and $n-\mathrm{C}_{5}$ is heavier than the heavy key (HHK). Each of these four designations exhibits a different type of composition profile curve as shown in Fig. 12. From the figure it is observed that liquid mole fraction of the light key $\left(n-\mathrm{C}_{4}\right)$ decreases linearly and continuously from the top of the column to the bottom.

The inverse occurs for the heavy key $\left(i-\mathrm{C}_{5}\right)$. Mole fractions of $\mathrm{C}_{3}$ (LLK) are almost constant over the rectifying section except near the top. Below the feed zone, C3 and $i$ $\mathrm{C}_{4}$ rapidly disappears from liquid stream. Similarly the concentration of $n-\mathrm{C}_{5}$, the HHK component increase from top to bottom.

\section{1) Comparison of distillate composition computed using inside out with other methods}

Fig. 13 presents comparison of distillation composition computed using different methods. The distillate composition obtained using these methods is almost same except for component 3 and 5 where the composition slightly deviates from composition obtained using other methods.

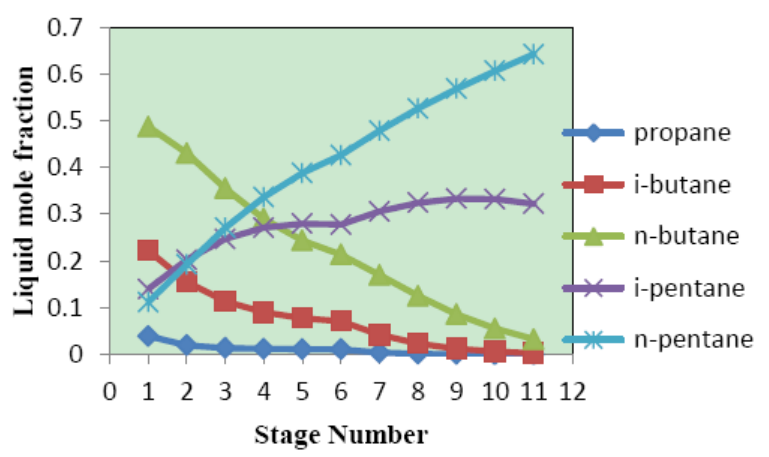

Fig. 12. Liquid composition profile computed using inside out method.

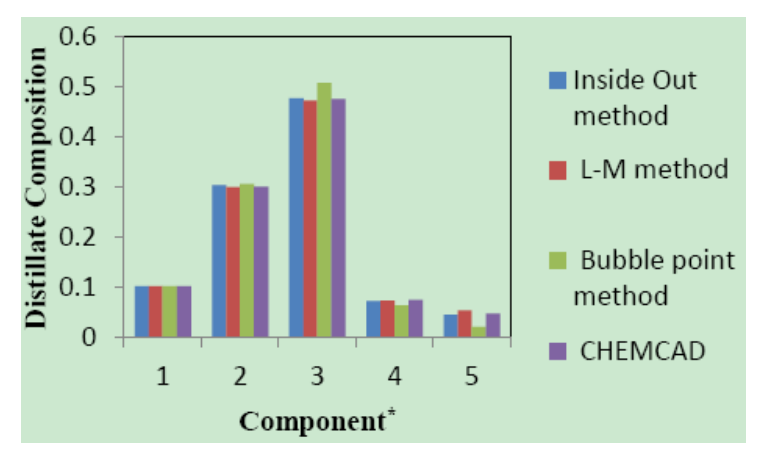

Fig. 13. Comparison of distillate composition computed using different methods.

${ }^{*}$ 1. Propane; 2. I-butane; 3- N-butane; 4. I-pentane; 5. N-pentane.

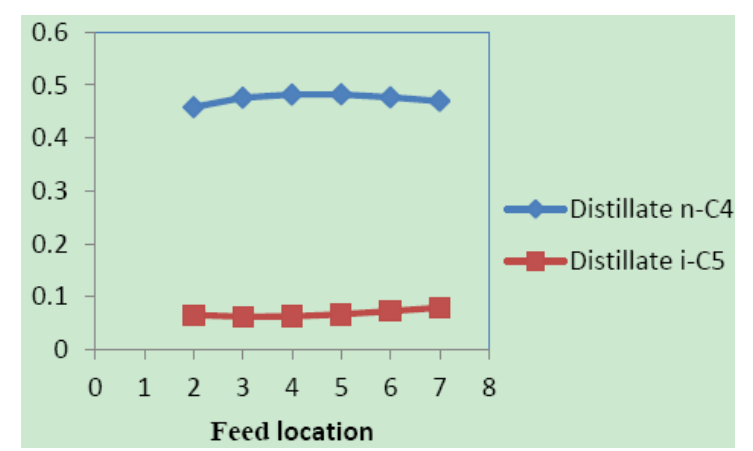

Fig. 14. Effect of feed location on distillate composition computed using inside out method.

\section{2) Effect of feed location on distillate and bottom product composition}

The Fig. 14 and Fig. 15 present the variation of LK and HK concentrations in distillate and residue respectively with the changes in feed stage location.

It is noted that feed location between stages 4 and 6 is effective in achieving the desired split between LK and HK. Beyond these stage numbers the separation is ineffective. Therefore appropriate feed stage location can be selected according to the composition desired.

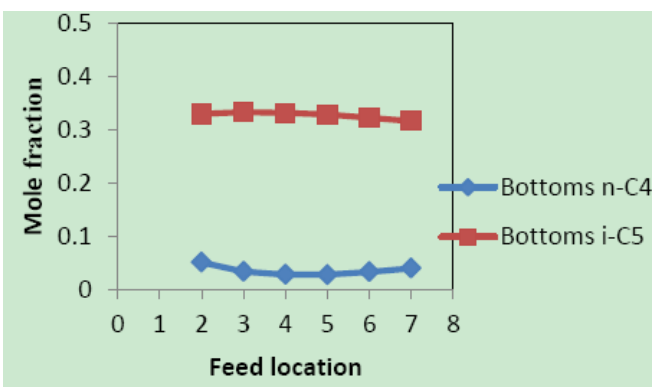

Fig. 15. Effect of feed location on bottom product composition computed using inside out method.

\section{E. Condenser and Reboiler Duty Estimation}

The condenser and reboiler duties for the column under study have been estimated and are $2.55 \times 10^{6} \mathrm{~J} / \mathrm{h}$ and $3.53 \times$ $10^{6} \mathrm{~J} / \mathrm{h}$ respectively.

\section{CONCLUSION}

The Performance analysis of distillation column for separation of multicomponent mixture has been carried out using equilibrium based (inside out) method and the results have been compared with the results obtained using Lewis Matheson method, bubble point method and using an SCDS column in CHEMCAD process simulator. From the analysis, it is observed that the results obtained using the inside out method are in close agreement with Lewis Matheson method and CHEMCAD. It is further observed that inside out method is just as efficient as methods like Lewis-Matheson for simple columns but is more robust for complicated columns where Lewis-Matheson method fails to converge. Also the simultaneous correction techniques used by SCDS column in CHEMCAD has a greater convergence time compared to inside out method. The bubble point method failed to provide as accurate results as the inside out method and hence it can be said that the inside out is a better alternative to bubble point method. Finally it is concluded that better convergence time, accurate results and the ability to solve complicated columns make inside out method the preferred choice for multicomponent distillation operation.

\section{NOMENCLATURE}

$\alpha_{m, n}=$ Relative volatility with respect to a base/reference component

$\epsilon=$ Damping factor

$F_{n}=$ Feed flow rate at stage $n$

$\overline{F_{m}}=$ Energy balance function/ Specification function to be converged using Newton Raphson

$f_{m, n}=$ Component feed flow rate at stage $n$ 
$h_{L}=$ Real enthalpy for liquid

$h_{V}=$ Real enthalpy for vapor

$h_{F}=$ Enthalpy of feed

$K_{m, n}=$ Liquid vapor distribution ratio for component $\mathrm{m}$ at stage $n$

$K_{b, n}=$ Base component liquid vapor distribution ratio at stage $n$

$l_{m, n}=$ Component liquid flow rate at stage $n$

$L_{n}=$ Liquid flow rate at stage $n$

$N=$ Total number of equilibrium stages

$Q_{n}=$ Heat transfer rate at stage $n$

$R_{L_{n}}=$ Liquid side stream withdrawal factor

$R_{v_{n}}=$ Vapor side stream withdrawal factor

$S_{m, n}=$ Stripping factor for component $\mathrm{m}$ at stage $n$

$S_{b, n}=$ Base stripping factor at stage $n$

$T_{n}=$ Temperature at stage $n$

$U_{n}=$ Liquid side stream flow rate at stage $n$

$V_{n}=$ Vapor flow rate at stage $n$

$v_{m, n}=$ Vapor component flow rate at stage $n$

$W_{n}=$ Vapor side stream flow rate at stage $n$

$w_{m, n}=$ weighing factor to be used in equation

$x_{m, n}=$ mole faction of liquid component $\mathrm{m}$ at stage $n$

$x=$ Iteration variable (Stripping factor/ Side stream factor)

used to converge $\overline{F_{m}}$

$\Delta x=$ Correction to the iteration variable

$y_{m, n}=$ mole faction of vapor component $\mathrm{m}$ at stage $n$

\section{REFERENCES}

[1] W. K. Lewis, and G. L. Matheson "Studies in distillation," Ind. Eng. Chem., vol. 24, pp. 494-498, May 1932.

[2] E. W. Thiele, and R. L. Geddes, "Computation Of Distillation Apparatus For Hydrocarbon Mixtures," Ind. Eng. Chem., vol. 25, pp. 289-295, March 1933

[3] J. R. Friday and B. D. Smith, "An analysis of the equilibrium stage separations problem - formulation and convergence," AIChE J., vol. 10, issue 5, pp. 698-707, Sep. 1964.

[4] J. F. Boston and S. L. Sullivan, Jr., "A new class of solution methods for multicomponent, multistage separation processes," Can. J. Chem. Engr., vol. 52, issue 1, pp. 52-63, Feb. 1974.

[5] L. M. Napthali and D. P. Sandholm, "Multicomponent separation calculations by linearization," AIChE Journal, vol. 17, issue 1, pp. 148-153, Jan. 1971.

[6] J. Simandl and W. Y. Svrcek, "Extension of the simultaneoussolution and inside-outside algorithms to distillation with chemical reactions," Computers and Chemical Engineering, vol. 15, issue 5, pp. 337-348, May 1991.

[7] J. F. Boston, "Inside-out algorithms for multicomponent separation process calculations," ACS Symposium Series No. 124, ch. 6, pp. 135-151, May 1980.

[8] R. A. Russell, "A flexible and reliable method solves single-tower and crude-distillation-column problems," Chem. Eng., vol. 90, issue 20, pp. 53-59, 1983.

[9] R. B. Saegar and P. R.Bishnoi, "A modified 'inside-out' algorithm for simulation of multistage multicomponent separation processes using the UNIFAC group-contribution method," Canadian Journal of Chemical Engineering, vol. 64, issue 5, pp. 759-767, Oct. 1986.

[10] J. Jelinek, "The calculation of multistage equilibrium separation problems with various specifications," Computational Chemical Engineering, vo. 12, pp. 195-198, 1988
[11] L. C. Shan Z. X. Ping, Z. S. Jiang, T. X. Shun, and X. S. Guang, "Simulation of multi-component multi-stage separation process - An improved algorithm and application," The Chinese Journal of Process Engineering, vol. 6, no. 2, pp. 247-254, April 2006.

[12] R. A. Trevino-Lozano, T. P. Kisala, and J. F. Boston, "A simplified absorber model for nonlinear simultaneous modular flow sheet calculations," Computers and Chemical Engineering, vol. 8, no. 2, pp. $105-115,1984$.

[13] J. C. Wang and G. E. Henke, "Tridiagonal matrix for distillation," Hydrocarbon Processing, vol. 45, no. 8, pp. 155-163, 1966.

[14] A. D. Sujata, "Absoiber stripper calculations made easier," Hydrocarbon Processing, vol. 40, no. 12, pp. 137-140, 1961.

[15] D. S. Buford, Design of Equilibrium Stage Processes, McGraw Hill Publication, pp. 278-279, 1963.

[16] H. Z. Kister, Distillation Design, McGraw-Hill, Inc, New York, 1992, ch. 4, p. 173.

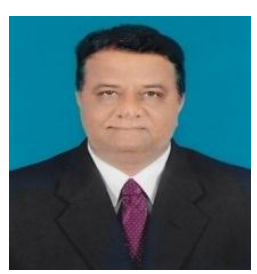

S. D. Manjare was born in Nasik, Maharashtra, on $1^{\text {st }}$ June, 1968. He completed his Ph.D. in the field of adsorption, the separation process from BITS Pilani, Rajasthan, India in 2004. Presently he works in the area of separation of multicomponent mixtures using distillation and absorption. He also works in the area of fuel cell and environmental engineering.

He has been working in BITS Pilani since 1997. He is actively involved in teaching, research and administration. Before joining BITS Pilani Rajasthan, he was a lecturer in the Department of Chemical Engineering in Pune University. He has taught several chemical engineering courses, for example, mass transfer operations, advanced separation processes, computational transport phenomenon, process design decisions, environmental pollution control, environmental management systems etc. He has guided several undergraduate and postgraduate students in the areas of fuel cell, pollution control, separation processes etc. Prof. Manjare has published several research papers in national, international journals and conferences of repute. He has conducted workshop on environmental management systems and auditing for the Engineering College teachers. He has completed five research projects in various areas like, adsorption, environmental management and fuel cell. He was instrumental in setting up of environmental pollution control laboratory in the Department of Chemical Engineering at BITS Pilani, Rajasthan. Among the research projects completed, the life cycle assessment of the Diammonium Phosphate (DAP) production at Zuari Industry Goa and IFFCO Kandla, Gujarat, is worth mentioning here.

Prof. Manjare is a member of AIChE and life member of Indian Institute of Chemical Engineering (IIChE). He is also a member of Hydrogen Association of India.

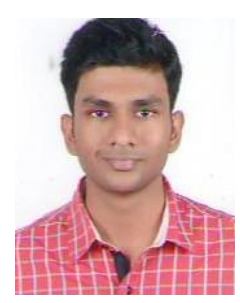

Shashwat Bansal was born in Patna, Bihar on $11^{\text {th }}$ March, 1993. He completed his B.E (Hons.) in chemical engineering from BITS Goa in June 2015. Presently he is working in the area of designing and simulating distillation columns for separating multicomponent mixtures.

He has pursued his undergraduate study from BITS Goa during 2011-2015. During the period he has completed internships in Essar power and Reliance Industries Limited and worked on academic projects in the field of molecular simulations, process design and development and multicomponent distillation and absorption. Presently he is working with UOP India Private Limited (a Honeywell company) in the field services division. 